\title{
NON-LIPSCHITZ DIFFERENTIABLE FUNCTIONS ON SLIT DOMAINS
}

\author{
L. BERNAL-GONZÁLEZ*, P. JIMÉNEZ-RODRÍGUEZ ${ }^{* *}$, \\ G.A. MUÑOZ-FERNÁNDEZ ${ }^{* *}$, AND J.B. SEOANE-SEPÚLVEDA**
}

\begin{abstract}
It is proved the existence of large algebraic structures -including large vector subspaces or infinitely generated free algebrasinside the family of non-Lipschitz differentiable real functions with bounded gradient defined on special non-convex plane domains. In particular, this yields that there are many differentiable functions on plane domains that do not satisfy the Mean Value Theorem.
\end{abstract}

\section{INTRODUCTION}

A standard result from Real Analysis states that, for any interval $I$ of the real line $\mathbb{R}$, a differentiable function $f: I \longrightarrow \mathbb{R}$ is Lipschitz if and only if it has bounded derivative. Recall that a mapping $\varphi:\left(X, d_{1}\right) \rightarrow\left(Y, d_{2}\right)$ between two metric spaces is called Lipschitz whenever there is a constant $k \in(0,+\infty)$ such that $d_{2}(\varphi(P), \varphi(Q)) \leq k d_{1}(P, Q)$ for all $P, Q \in X$. One could think if the above mentioned result still holds for domains of several variables. If $N \in \mathbb{N}:=\{1,2,3, \ldots\}$, by a domain of $\mathbb{R}^{N}$ we mean a nonempty connected open subset $\Omega \subset \mathbb{R}^{N}$. If $\Omega$ is a convex domain, a simple application of the Mean Value Theorem yields that every differentiable function $f: \Omega \rightarrow \mathbb{R}$ with bounded gradient is Lipschitz. This statement does not hold if $\Omega$ is not convex. In fact, Jiménez, Muñoz and Seoane [11, Theorem 3.2] showed in 2012 that even in the case of the (very simple, nonconvex) domain of $\mathbb{R}^{2}$ given by

$$
D=\left\{(x, y) \in \mathbb{R}^{2}: x^{2}+y^{2}<1\right\} \backslash\left\{(x, y) \in \mathbb{R}^{2}: x=0 \text { and } y \geq 0\right\}
$$

there is a plethora of such non-Lipschitz functions. Their result reads as follows. By $\mathfrak{c}$ we denote the cardinality of the continuum.

Theorem 1.1. The set of differentiable functions $f: D \rightarrow \mathbb{R}$ with bounded gradient, non-Lipschitz and therefore not satisfying the classical Mean Value Theorem contains, except for the zero function, a $\mathfrak{c}$-dimensional vector space.

2010 Mathematics Subject Classification. Primary 26B35; Secondary 15A03, 31C05.

Key words and phrases. Non-Lipschitz function, differentiable function, domain in the plane, free algebra.

${ }^{*}$ Supported by the Plan Andaluz de Investigacin de la Junta de Andaluca FQM-127 Grant P08-FQM-03543 and by MEC Grant MTM2015-65242-C2-1-P.

${ }^{* *}$ Supported by the Spanish Ministry of Science and Innovation, Grant MTM201234341. 
Our aim in this paper is to study the existence of such special functions on more general plane domains. In fact we will produce dense vector spaces as well as infinitely generated algebras consisting, except for zero, of these pathological objects. This will be accomplished in Section 4. In Section 2 some notation will be fixed, and a number of concepts about lineability will be recalled, while in Section 3 a positive (non-pathological) statement will be provided, so as to motivate the geometrical restrictions given in Section 4 for the corresponding domains.

\section{LinEABITY NOTIONS AND NOTATION}

We need some background about the modern topic of lineability, that nowadays has had a major influence in many different areas on mathematics. The goal of lineability is to describe the algebraic size of a set that not necessarily enjoys any algebraic structure. The concepts provided in the next paragraph where coined in $[3,5,9]$; see also the survey paper [7] and the book [1] for a source of notions and results.

If $X$ is a vector space, $\mu$ is a cardinal number and $A \subset X$, then $A$ is said to be lineable if there is an infinite dimensional vector space $M$ such that $M \backslash\{0\} \subset A$, and $\mu$-lineable if there exists a vector space $M$ with $\operatorname{dim}(M)=\mu$ and $M \backslash\{0\} \subset A$. If, in addition, $X$ is a topological vector space, then $A$ is said to be dense-lineable ( $\mu$-dense-lineable) in $X$ whenever there is a dense (a dense $\mu$-dimensional, resp.) vector subspace $M$ of $X$ satisfying $M \backslash\{0\} \subset A$. When $X$ is a topological vector space contained in some (linear) algebra then $A$ is called strongly $\mu$-algebrable if there exists a $\mu$-generated free algebra $M$ with $M \backslash\{0\} \subset A$. Note that if $X$ is contained in a commutative algebra then a set $B \subset X$ is a generating set of some free

algebra contained in $A$ if and only if for any $N \in \mathbb{N}$, any nonzero polynomial $P$ in $N$ variables without constant term and any distinct $f_{1}, \ldots, f_{N} \in B$, we have $P\left(f_{1}, \ldots, f_{N}\right) \neq 0$ and $P\left(f_{1}, \ldots, f_{N}\right) \in A$.

Under the above terminology, Theorem 1.1 tells us that the set described in it is c-lineable.

The next auxiliary, general result about lineability will be used later. Many variants of it have been proved, and it can be found in $[1,7]$ and the references contained therein.

Lemma 2.1. Assume that $X$ is a metrizable separable topological vector space. Let $A \subset X$ be $\mu$-lineable, where $\mu$ is an infinite cardinal number. Suppose that there exists a dense vector subspace $B \subset X$ such that $A+B \subset$ $A$ and $A \cap B=\varnothing$. Then $A$ is $\mu$-dense-lineable in $X$.

Remark 2.2. In connection with the Lipschitz property, additional results completing Theorem 1.1 can be found in [11], as for instance:

(a) The set of continuous functions on $[0,1]$ which are a.e. differentiable, with a.e. bounded derivative and not Lipschitz, is $\mathfrak{c}$-lineable. 
(b) The set of differentiable functions $f: \mathbb{R} \longrightarrow \mathbb{R}^{2}$ that do not enjoy the classical Mean Value theorem is $\mathfrak{c}$-lineable.

All three results (a), (b) and Theorem 1.1 can be improved to dense-lineability by means of Lemma 2.1. Concerning (a), see also [10].

Now, we recall some notions and fix some notation, most of it standard. By $|z|$ we represent the Euclidean norm $\left(z_{1}^{2}+\cdots+z_{n}^{2}\right)^{1 / 2}$ of the point $z=\left(z_{1}, \ldots, z_{N}\right) \in \mathbb{R}^{N}$. A domain in $\mathbb{R}^{N}$ is a nonempty connected open subset of $\mathbb{R}^{N}$. If $z$ and $w$ are points of a domain $\Omega \subset \mathbb{R}^{N}$ then an arc in $\Omega \subset \mathbb{R}^{N}$ joining $z$ to $w$ is a continuous function $\gamma:[0,1] \rightarrow \Omega$ with $\gamma(0)=z$ and $\gamma(1)=w$. The family of these arcs will be denoted by $\Gamma(z, w ; \Omega)$. The length of an arc $\gamma$ is the supremum of the lengths of all polygonal curves inscribed in it, that is,

$\operatorname{length}(\gamma)=\sup \left\{\sum_{j=1}^{p}\left|\gamma\left(t_{j}\right)-\gamma\left(t_{j-1}\right)\right|: 0=t_{0}<t_{1}<\cdots<t_{p}=1, p \in \mathbb{N}\right\}$

If $z_{0} \in \mathbb{R}^{N}$ then an arc joining $z_{0}$ to infinity is a continuous mapping $\gamma:[0,+\infty) \rightarrow \mathbb{R}^{N}$ such that $\lim _{t \rightarrow+\infty}|\gamma(t)|=+\infty$. By abuse of language, we will sometimes identify the arc $\gamma$ with its curve, that is, with the image $\Gamma=\gamma(I)(I=[0,1]$ or $[0,+\infty))$. A Jordan arc is an arc $\gamma$ without selfintersections, that is, the mapping $\gamma$ is one-to-one. A domain in $\mathbb{R}^{2}$ is said to be simply connected provided that its complement with respect to the one-point compactification of $\mathbb{R}^{2}$ (the extended plane) is connected.

Finally, the symbol $\mathcal{C}(\Omega)$ will stand for the vector space of all real continuous functions defined on a domain $\Omega$. It is well known that this space becomes a complete metrizable topological vector space when it is endowed with the topology of uniform convergence in compacta. Moreover, it is separable, and the polynomials form a dense subset of it. The space $\mathcal{C}_{c}^{\infty}(\Omega)$ of infinitely differentiable functions having compact support contained in $\Omega$ (the "test" functions) is also dense in $\mathcal{C}(\Omega)$.

\section{LIPSCHITZ PROPERTY AND CONVEXITY OF DOMAINS}

In view of the comments made in the Introduction, one might believe that every non-convex domain in $\mathbb{R}^{N}$ supports a non-Lipschitz differentiable function having bounded gradient. This is far from being true. In fact, the probability for finding such functions depends strongly on the structure of the domain. In this structure, convexity plays an essential but not determining role. We will show that if the non-convexity "is not extreme", such pathological functions are forbidden. In order to do this formally, we introduce the concept given in the next definition. 
Definition 3.1. Let $\Omega \subset \mathbb{R}^{N}$ be a domain. The modulus of non-convexity of $\Omega$ is defined as the number

$$
\omega_{n c}(\Omega)=\sup _{\substack{z, w \in \Omega \\ z \neq w}} \frac{\inf \{\operatorname{length}(\gamma): \gamma \in \Gamma(z, w ; \Omega)\}}{|z-w|} .
$$

Obviously, $\omega_{n c}(\Omega) \in[1,+\infty]$, and if $\Omega$ is convex then $\omega_{n c}(\Omega)=1$. The reverse is not true because, for instance, a convex domain with one point removed has modulus equal to 1 . The domain $D$ of Theorem 1.1 satisfies $\omega_{n c}(D)=+\infty$; indeed, there are pairs of points in $D$ that are arbitrarily close in $\mathbb{R}^{2}$ but being far from each other when "walking" along $D$. As an intermediate example, consider, for given $\alpha \in(0, \pi / 2)$, the non-convex domain

$$
\Omega_{\alpha}=\left\{r e^{i \theta}: 0<r<1 \text { and } \alpha<\theta<2 \pi-\alpha\right\},
$$

where we have identified $\mathbb{R}^{2}$ and the complex plane $\mathbb{C}$ in the usual way, so that their elements can be written as $z=(x, y)=x+i y=r e^{i \theta}$. Elementary trigonometrical arguments lead us to

$$
1<\omega_{n c}\left(\Omega_{\alpha}\right) \leq \sec \alpha+\operatorname{cosec} \alpha+\operatorname{cosec} \alpha \operatorname{cotan} \alpha .
$$

Proposition 3.2. Let $\Omega \subset \mathbb{R}^{N}$ be a domain satisfying $\omega_{n c}(\Omega)<+\infty$. Then any differentiable function $\Omega \rightarrow \mathbb{R}$ with bounded gradient is Lipschitz.

Proof. By hypothesis, there exists a constant $C \in(0,+\infty)$ such that, for every pair of distinct points $z, w \in \Omega$ and every $\varepsilon>0$, there is an arc $\gamma \in \Gamma(z, w ; \Omega)$ satisfying length $(\gamma) \leq C|z-w|+\varepsilon$. Choosing $\varepsilon=|z-w|$, we get an arc $\gamma \in \Gamma(z, w ; \Omega)$ with length $(\gamma) \leq(C+1)|z-w|$. Since the curve $\gamma([0,1])$ is a compact subset of $\Omega$, one has $\rho:=\operatorname{dist}\left(\gamma([0,1]), \mathbb{R}^{N} \backslash \Omega\right)>0$. Because of the uniform continuity of $\gamma$ on the compact set $[0,1]$, there exists a $\delta>0$ such that $|\gamma(u)-\gamma(v)|<\rho$ provided that $u, v$ are points of $[0,1]$ with $|u-v|<\delta$. Take $p \in \mathbb{N}$ such that $1 / p<\delta$, and let $t_{j}=j / p$ $(j=0,1, \ldots, p)$. The selection of these points guarantees that each segment $\left[\gamma\left(t_{j-1}\right), \gamma\left(t_{j}\right)\right]$ remains in $\Omega$, so that we can apply the Mean Value Theorem to the points $\gamma\left(t_{j-1}\right), \gamma\left(t_{j}\right)$ for any differentiable function $\Omega \rightarrow \mathbb{R}$.

Assume that $f$ is one of such functions and that, in addition, $\operatorname{grad} f$ is bounded. Then there is $\alpha \in(0,+\infty)$ such that $|\operatorname{grad} f(\xi)| \leq \alpha$ for all $\xi \in \Omega$. Due to the Main Value Theorem and the triangle inequality, we obtain for certain $\xi_{j} \in\left[\gamma\left(t_{j-1}\right), \gamma\left(t_{j}\right)\right](j=1, \ldots, p)$ that

$$
\begin{aligned}
|f(z)-f(w)| & \leq \sum_{j=1}^{p}\left|f\left(\gamma\left(t_{j}\right)\right)-f\left(\gamma\left(t_{j-1}\right)\right)\right| \leq \sum_{j=1}^{p}\left|\operatorname{grad} f\left(\xi_{j}\right)\right|\left|\gamma\left(t_{j}\right)-\gamma\left(t_{j-1}\right)\right| \\
& \leq \alpha \sum_{j=1}^{p}\left|\gamma\left(t_{j}\right)-\gamma\left(t_{j-1}\right)\right| \leq \alpha \operatorname{length}(\gamma) \leq \alpha(C+1)|z-w|,
\end{aligned}
$$

which shows that $f$ is Lipschitz (with constant $k=\alpha(C+1)$ ). 


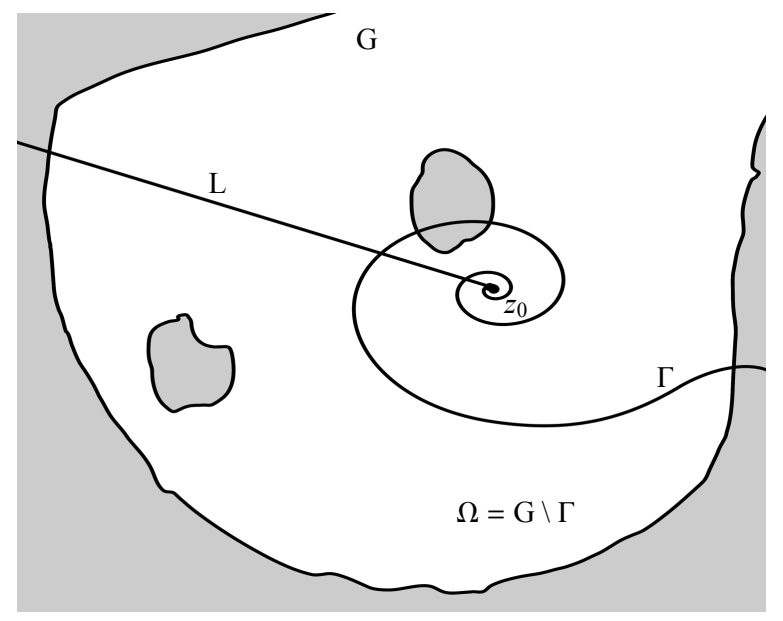

FiguRE 1. A slit domain $\Omega$.

As a consequence, it seems that, in order to obtain non-Lipschitz differentiable functions with bounded gradient, the complement of $\Omega$ must be very "thin" somewhere. This motivates the introduction of the special domains considered in the next section.

\section{Slit DOMAINS AND NON-Lipschitz FUnCTIONS With BOUNDED GRADIENT}

In this section, we restrict ourselves to the case $N=2$, that is, our domains will live in $\mathbb{R}^{2}=\mathbb{C}$. This is illustrative enough because, simply by fixing $N-2$ variables, we obtain immediately examples of domains in $\mathbb{R}^{N}$ $(N \geq 3)$ supporting non-Lipschitz differentiable functions.

Definition 4.1. A domain $\Omega \subset \mathbb{R}^{2}$ is said to be a slit domain (see Figure 1) whenever there exist a domain $G \subset \mathbb{R}^{2}$, a point $z_{0} \in G$, a Jordan arc $\Gamma$ joining $z_{0}$ to infinity, and a ray $L$ starting from $z_{0}$, such that $\Omega=G \backslash \Gamma$ and the set $\Gamma \cap L \cap G$ is finite.

As simple instances, $\mathbb{C} \backslash[0,+\infty)$ is a slit domain and $D$ (see Introduction) is a bounded slit domain. But note that if $\Gamma$ is the spiral $\rho=\theta$ (in polar coordinates) then $\mathbb{C} \backslash \Gamma$ is not a slit domain.

Theorem 4.2. Let $\Omega \subset \mathbb{R}^{2}$ be a slit domain. Then the following holds:

(a) The set of non-Lipschitz bounded continuously differentiable functions $\Omega \rightarrow \mathbb{R}$ having bounded gradient is $\mathfrak{c}$-dense-lineable in $\mathcal{C}(\Omega)$ and strongly $\mathfrak{c}$-algebrable.

(b) If, in addition, $\Omega$ is bounded, the set of non-Lipschitz bounded realanalytic functions $\Omega \rightarrow \mathbb{R}$ having bounded gradient is dense-lineable.

Proof. (a) There exist a domain $G$, a point $z_{0}$, an unbounded Jordan arc $\Gamma$ and a ray $L$ satisfying the conditions in Definition 4.1. Without loss of 
generality, we may assume that $z_{0}=0$. Since $\Gamma$ is a Jordan arc joining 0 to $\infty$, its complement $\mathbb{C} \backslash \Gamma$ is a simply connected domain not containing the origin. Therefore (see, e.g., [13]) there is a continuous branch $\varphi(z)$ of $\arg z$ in $\mathbb{C} \backslash \Gamma$, which in turn is the imaginary part of a continuous branch $\psi$ of $\log z$ in $\mathbb{C} \backslash \Gamma$. Hence $\varphi$ is harmonic, so continuously differentiable as a function of $(x, y)$. In addition, the Cauchy-Riemann equations as applied to $\psi(x, y)=\frac{1}{2} \ln \left(x^{2}+y^{2}\right)+i \varphi(x, y)$ yield

$$
\frac{\partial \varphi}{\partial x}(z)=\frac{-y}{x^{2}+y^{2}}=\frac{-y}{|z|^{2}} \quad \text { and } \quad \frac{\partial \varphi}{\partial y}(x, y)=\frac{x}{x^{2}+y^{2}}=\frac{x}{|z|^{2}} \quad(z=(x, y)) .
$$

Furthermore, we have that $G \cap L \cap \Gamma$ is finite, say card $(G \cap L \cap \Gamma)=N$. Then it is easy to see that if an arc joins any two points inside $\Omega=\mathbb{C} \backslash \Gamma$ then the argument of a variable point along such an arc cannot increase (or decrease) continuously more than $2 \pi(N+1)$. In other words, the amplitude of the interval given by the image of $G$ under $\varphi$ is less than $2 \pi(N+1)$, so $\varphi$ is bounded on $G$. By adding an appropriate $2 \pi m(m \in \mathbb{N})$, we may even assume that $\varphi(z)>0$ for all $z \in G$.

Next, we focus on the origin $z_{0}=0$ of $\Gamma$. Take $R>0$ such that $z \in G$ whenever $|z|<R$. Let $\gamma:[0,+\infty) \rightarrow \mathbb{C}$ be a parametrization of $\Gamma$, so that $\gamma$ is one-to-one, $\gamma(0)=0$ and $|\gamma(t)| \rightarrow+\infty$ as $t \rightarrow+\infty$. The continuity of $\gamma$ together with the fact that $|\gamma(t)| \rightarrow+\infty$ as $t \rightarrow+\infty$ entails the existence, for given $\varepsilon, \widetilde{\varepsilon}$ with $0<\widetilde{\varepsilon}<\varepsilon<R$, of at least one point $\xi \in \Gamma \backslash\{0\}$ such that $\widetilde{\varepsilon}<|\xi|<\varepsilon$. If $\delta_{1}:=\min \{|\xi|-\widetilde{\varepsilon}, \varepsilon-|\xi|\}$ then the open disc $\left\{|z-\xi|<\delta_{1}\right\}$ is contained in $G$. Still it may be the case that there is no need to surround 0 in order to connect two given points of $\left\{|z-\xi|<\delta_{1}\right\} \backslash \Gamma$ belonging to two different components of this set. In order to bridge this problem, let $\Gamma_{1}$ be the connected component (in $\left\{|z-\xi|<\delta_{1}\right\}$ ) of $\Gamma \cap\left\{|z-\xi|<\delta_{1}\right\}$ containing $\xi$. Then $\Gamma_{1}$ is the (bijective) image under $\gamma$ of an interval $(a, b) \subset(0,+\infty)$, so that $\xi=\gamma(c)$ for a unique $c \in(a, b)$. Since $\Gamma$ is a Jordan arc tending to infinity, the set $\Gamma_{2}:=\gamma([0, a] \cup[b,+\infty))=\Gamma \backslash \Gamma_{1}$ is closed in $\mathbb{C}$ and it does not contain the point $\xi$. Therefore $\operatorname{dist}\left(\xi, \Gamma_{2}\right)>0$. Let

$$
\delta_{0}:=\min \left\{\delta_{1}, \operatorname{dist}\left(\xi, \Gamma_{2}\right)\right\}>0 .
$$

For each $\delta \in\left(0, \delta_{0}\right)$, the set $\{|z-\xi|<\delta\} \cap \Gamma$ is contained in $\Gamma_{1}$ and, by construction, there are points $u, v$ (belonging to certain two different components of the "old" set $\left.\left\{|z-\xi|<\delta_{1}\right\} \backslash \Gamma\right)$ satisfying $|u-\xi|<\delta,|v-\xi|<$ $\delta$, and for which one needs to surround 0 in order to connect them inside $G$ without crossing $\Gamma$. Moreover, taking $\delta$ small enough, we can make the increment of $\arg z$ as close to $2 \pi$ (or to $2 \pi N$, for some $N \in \mathbb{N}$ ) as desired when $z$ goes from $u$ up to $v$ inside $\Omega$. Altogether, we conclude with the help of the triangle inequality the following assertion:

(A) Given $\varepsilon, \widetilde{\varepsilon}$ with $0<\widetilde{\varepsilon}<\varepsilon<R$ and $\delta>0$, there are $u, v \in \Omega$ such that $\widetilde{\varepsilon}<|u|<\varepsilon, \widetilde{\varepsilon}<|v|<\varepsilon,|u-v|<\delta$ and $|\varphi(u)-\varphi(v)| \geq 1$. 
Let us consider, for every $c \in(1,2)$, the function $f_{c}: \Omega \rightarrow \mathbb{R}$ given by

$$
f_{c}(z)=\frac{|z|^{c}}{1+|z|^{2}} \varphi(z)
$$

From the boundedness of $\varphi$ (say, $|\varphi(z)| \leq M$ on $\Omega$ ), it follows that $\left|f_{c}(z)\right|$ is not greater than $\frac{M|z|^{c}}{1+|z|^{2}}$, and this function is continuous on $\mathbb{C}$ and tends to 0 as $z \rightarrow \infty$ (because $0<c<2$ ). Hence it is bounded on $\mathbb{C}$, so $f_{c}$ is bounded on $\Omega$. Now, simple calculations give

$$
\begin{aligned}
& \frac{\partial f_{c}}{\partial x}(z)=\frac{c x|z|^{2 c-1}-2 x|z|^{c}}{\left(1+|z|^{2}\right)^{2}} \varphi(z)+\frac{-y}{|z|^{2}} \frac{|z|^{c}}{1+|z|^{2}} \text { and } \\
& \frac{\partial f_{c}}{\partial y}(z)=\frac{c y|z|^{2 c-1}-2 y|z|^{c}}{\left(1+|z|^{2}\right)^{2}} \varphi(z)+\frac{x}{|z|^{2}} \frac{|z|^{c}}{1+|z|^{2}},
\end{aligned}
$$

which are continuous on $\Omega$ because $\varphi$ is continuous and $0 \notin \Omega$. Therefore every $f_{c}$ is a bounded continuously differentiable function. Moreover, from the fact $|x|,|y| \leq|z|$, it follows that both $\left|\frac{\partial f_{c}}{\partial x}\right|$ and $\left|\frac{\partial f_{c}}{\partial y}\right|$ are not greater than $\frac{c|z|^{2 c}+2|z|^{c+1}}{\left(1+|z|^{2}\right)^{2}} M+\frac{|z|^{c-1}}{1+|z|^{2}}$. The last function is continuous on $\mathbb{C}$ and tends to 0 as $z \rightarrow \infty$ because $0<2 c<4,0<c+1<4$ and $0<c-1<2$. Thus, it is bounded, which entails the boundedness of $\operatorname{grad} f_{c}$ on $\Omega$.

Now, fix a set $H \subset(1,2)$ with $\operatorname{card}(H)=\mathfrak{c}$ and linearly independent over the field $\mathbb{Q}$ of rational numbers. Let us consider the algebra $\mathcal{A}$ generated by the functions $f_{c}(c \in H)$. Trivially, $\mathcal{A}$ consists of bounded continuously differentiable functions $\Omega \rightarrow \mathbb{R}$ having bounded gradient. It should be shown that $\mathcal{A}$ is freely generated by the $f_{c}$ 's and that each nonzero member of $\mathcal{A}$ is non-Lipschitz on $\Omega$. Since $\operatorname{card}(H)=\mathfrak{c}$ and, trivially, any nonLipschitz function is not identically zero, it is enough to prove the following:

(B) Given $p \in \mathbb{N}$, a non-identically zero polynomial $P$ of $p$ real variables without constant term and different reals $c_{1}, \ldots, c_{p} \in H$, the function $F:=P\left(f_{c_{1}}, \ldots, f_{c_{p}}\right)$ is non-Lipschitz on $\Omega$.

In order to prove $(\mathrm{B})$, a little more notation is needed. We set $\mathbb{N}_{0}=$ $\mathbb{N} \cup\{0\}$. If $p \in \mathbb{N}$ then each element of $[0,+\infty)^{p}$ (in particular, each element of $\left.\mathbb{N}_{0}^{p}\right)$ has the form $\mathbf{c}=\left(c_{1}, \ldots, c_{p}\right)$ with $c_{j} \geq 0$ for all $j$. We let $|\mathbf{c}|=c_{1}+\cdots+c_{p}$. For $\mathbf{u}, \mathbf{v} \in[0,+\infty)^{p}$, we adopt the notation $\mathbf{u v}=$ $\left(u_{1} v_{1}, \ldots, u_{p} v_{p}\right)$, so that $|\mathbf{u v}|=u_{1} v_{1}+\cdots+u_{p} v_{p}$.

Fix a function $F=P\left(f_{c_{1}}, \ldots, f_{c_{p}}\right)$ as in (B). Then there is a nonempty subset $J \subset \mathbb{N}_{0}^{p} \backslash\{(0, \ldots, 0)\}$ as well as nonzero reals $\alpha_{\mathbf{m}}(\mathbf{m} \in J)$ such that

$$
F(z)=\sum_{\mathbf{m} \in J} \alpha_{\mathbf{m}} \frac{|z|^{|\mathbf{m c}|}}{\left(1+|z|^{2}\right)^{|\mathbf{m}|}} \cdot \varphi(z)^{|\mathbf{m}|} \text { for all } z \in \Omega .
$$


Due to the linear $\mathbb{Q}$-independence of $c_{1}, \ldots, c_{p}$, the numbers $|\mathbf{m c}|(\mathbf{m} \in J)$ are pairwise different. There is a unique $\mathbf{n} \in J$ such that $|\mathbf{n c}|=\min \{|\mathbf{m c}|$ : $\mathbf{m} \in J\}$. For every pair of points $u, v \in \Omega$ we can write

$$
\begin{aligned}
& F(u)-F(v)=\alpha_{\mathbf{n}}\left(\frac{|u|^{|\mathbf{n c}|}}{\left(1+|u|^{2}\right)^{|\mathbf{n}|}} \cdot \varphi(u)^{|\mathbf{n}|}-\frac{|v|^{|\mathbf{n c}|}}{\left(1+|v|^{2}\right)^{|\mathbf{n}|}} \cdot \varphi(v)^{|\mathbf{n}|}\right) \\
& +\sum_{\mathbf{m} \in J \backslash\{\mathbf{n}\}} \alpha_{\mathbf{m}}\left(\frac{|u|^{|\mathbf{m c}|}}{\left(1+|u|^{2}\right)^{|\mathbf{m}|}} \cdot \varphi(u)^{|\mathbf{m}|}-\frac{|v|^{|\mathbf{m} \mathbf{c}|}}{\left(1+|v|^{2}\right)^{|\mathbf{m}|}} \cdot \varphi(v)^{|\mathbf{m}|}\right) \\
& =\frac{\alpha_{\mathbf{n}}|u|^{|\mathbf{n c}|}}{\left(1+|u|^{2}\right)^{|\mathbf{n}|}} \cdot\left(\varphi(u)^{|\mathbf{n}|}-\varphi(v)^{|\mathbf{n}|}\right) \\
& +\alpha_{\mathbf{n}}\left(\frac{|u|^{|\mathbf{n c}|}}{\left(1+|u|^{2}\right)^{|\mathbf{n}|}}-\frac{|v|^{|\mathbf{n c}|}}{\left(1+|v|^{2}\right)^{|\mathbf{n}|}}\right) \cdot \varphi(v)^{|\mathbf{n}|} \\
& +\sum_{\mathbf{m} \in J \backslash\{\mathbf{n}\}} \alpha_{\mathbf{m}}\left(\frac{|u|^{|\mathbf{m c}|}}{\left(1+|u|^{2}\right)^{|\mathbf{m}|}} \cdot \varphi(u)^{|\mathbf{m}|}-\frac{|v|^{|\mathbf{m c}|}}{\left(1+|v|^{2}\right)^{|\mathbf{m}|}} \cdot \varphi(v)^{|\mathbf{m}|}\right) \\
& =: S_{1}(u, v)+S_{2}(u, v)+S_{3}(u, v) \text {. }
\end{aligned}
$$

Our final goal is to prove the following: given $K \in(0,+\infty)$, there are two points $u, v \in \Omega$ such that $|F(u)-F(v)|>K|u-v|$.

Before going on, an elementary inequality is needed. Namely, if $a, b \in$ $(0,+\infty)$ and $|a-b| \geq 1$ then $\left|a^{m}-b^{m}\right| \geq 1$ for all $m \in \mathbb{N}$. Indeed, if $a>b$, say, then $a \geq 1$. It follows that $\left|a^{m}-b^{m}\right|=a^{m}-b^{m}=(a-b)\left(a^{m-1}+\right.$ $\left.a^{m-2} b+a^{m-3} b^{2}+\cdots+b^{m-1}\right) \geq(a-b) a^{m-1} \geq 1 \cdot 1=1$.

Recall that $\varphi$ is positive and bounded on $\Omega$. Fix $K \in(0,+\infty)$. Let us denote

$$
\begin{gathered}
\alpha:=2 \max \left\{\left(\sup _{z \in \Omega} \varphi(z)\right)^{|\mathbf{m}|}: \mathbf{m} \in J\right\} \sum_{\mathbf{m} \in J}\left|\alpha_{\mathbf{m}}\right| \\
\gamma:=\min \{|\mathbf{m c}|-|\mathbf{n c}|: \mathbf{m} \in J \backslash\{\mathbf{n c}\}\} .
\end{gathered}
$$

Note that $\gamma>0$. Fix an $\varepsilon>0$ with

$$
\varepsilon<\min \left\{1, R,\left(\frac{\left|\alpha_{\mathbf{n}}\right|}{2^{|\mathbf{n}|+2} \alpha}\right)^{1 / \gamma}\right\} .
$$

From (3) one derives that $\alpha \varepsilon^{\gamma} 2^{|\mathbf{n}|+2} /\left|\alpha_{\mathbf{n}}\right|<1$. Choose any $\beta$ with

$$
\left(\frac{\alpha \varepsilon^{\gamma} 2^{|\mathbf{n}|+2}}{\left|\alpha_{\mathbf{n}}\right|}\right)^{\frac{1}{|\mathbf{n c}|}}<\beta<1 .
$$

Define $\widetilde{\varepsilon}:=\beta \varepsilon$. Then $0<\widetilde{\varepsilon}<\varepsilon$. Now, the uniform continuity of the function $z \mapsto \frac{|z|^{|\mathbf{n c}|}}{\left(1+|z|^{2}\right)^{|\mathbf{n}|}}$ on the compact disc $\{|z| \leq \varepsilon\}$ entails the existence 
of a $\delta>0$ such that

$$
\left|\frac{|u|^{|\mathbf{n c}|}}{\left(1+|u|^{2}\right)^{|\mathbf{n}|}}-\frac{|v|^{|\mathbf{n c}|}}{\left(1+|v|^{2}\right)^{|\mathbf{n}|}}\right|<\frac{\widetilde{\varepsilon}^{|\mathbf{n c}|}}{2^{|\mathbf{n}|+1}\left(\sup _{z \in \Omega} \varphi(z)\right)^{|\mathbf{n}|}}
$$

whenever $|u|,|v|<\varepsilon$ and $|u-v|<\delta$. We can select such a $\delta>0$ to satisfy, in addition,

$$
\delta<\frac{\left|\alpha_{\mathbf{n}}\right| \widetilde{\varepsilon}^{\mid \mathbf{n c}} \mid}{2^{|\mathbf{n}|+2} K}
$$

From the triangle inequality, we obtain

$$
|F(u)-F(v)| \geq\left|S_{1}(u, v)\right|-\left|S_{2}(u, v)\right|-\left|S_{3}(u, v)\right| \text { for all } u, v \in \Omega .
$$

According to $(\mathrm{A})$, there are $u, v \in \Omega$ such that $\widetilde{\varepsilon}<|u|<\varepsilon, \widetilde{\varepsilon}<|v|<\varepsilon$, $|u-v|<\delta$ and $|\varphi(u)-\varphi(v)| \geq 1$. Then $\left|\varphi(u)^{|\mathbf{n}|}-\varphi(v)^{|\mathbf{n}|}\right| \geq 1$. Since $\widetilde{\varepsilon}<|u|<\varepsilon<1$, we get

$$
\left|S_{1}(u, v)\right| \geq \frac{\left|\alpha_{\mathbf{n}}\right||u|^{|\mathbf{n c}|}}{\left(1+|u|^{2}\right)^{|\mathbf{n}|}} \cdot 1 \geq \frac{\left|\alpha_{\mathbf{n}}\right| \widetilde{\varepsilon}^{|\mathbf{n c}|}}{2^{|\mathbf{n}|}} .
$$

From (5), it follows that

$$
\left|S_{2}(u, v)\right| \leq \frac{\left|\alpha_{\mathbf{n}}\right| \widetilde{\varepsilon}^{|\mathbf{n c}|}}{2^{|\mathbf{n}|+1}}
$$

Now, as a consequence of (1), (2) and the fact $\varepsilon<1$ (note that $\varepsilon^{|\mathbf{m}|}=$ $\varepsilon^{|\mathbf{m c}|-|\mathbf{n c}|} \varepsilon^{|\mathbf{n c}|} \leq \varepsilon^{\gamma} \varepsilon^{|\mathbf{n c}|}$ if $\left.\mathbf{m} \neq \mathbf{n}\right)$, we derive

$$
\left|S_{3}(u, v)\right| \leq \alpha \varepsilon^{\gamma} \varepsilon^{|\mathbf{n c}|} .
$$

Finally, from (4), (6), (7), (8) and (9), we can conclude

$$
\begin{aligned}
|F(u)-F(v)| & \geq \frac{\left|\alpha_{\mathbf{n}}\right| \widetilde{\varepsilon}^{|\mathbf{n c}|}}{2^{|\mathbf{n}|}}-\frac{\left|\alpha_{\mathbf{n}}\right| \widetilde{\varepsilon}^{|\mathbf{n c}|}}{2^{|\mathbf{n}|+1}}-\alpha \varepsilon^{\gamma} \varepsilon^{|\mathbf{n c}|} \\
& \geq \frac{\left|\alpha_{\mathbf{n}}\right| \beta^{|\mathbf{n c}|} \varepsilon \varepsilon^{|\mathbf{n c}|}}{2^{|\mathbf{n}|+1}}-\frac{\left|\alpha_{\mathbf{n}}\right| \beta^{|\mathbf{n c}|} \varepsilon^{|\mathbf{n c}|}}{2^{|\mathbf{n}|+2}} \\
& =\frac{\left|\alpha_{\mathbf{n}}\right| \beta^{|\mathbf{n c}|} \varepsilon}{2^{|\mathbf{n}|+2}}>K \delta>K|u-v|,
\end{aligned}
$$

as required. The strong $\mathfrak{c}$-algebrability has been proved.

As for the $\mathfrak{c}$-dense-lineability, note that the algebra $\mathcal{A}$ above is, of course, a $\mathfrak{c}$-dimensional vector space. Hence the family $A$ consisting of all functions having the properties given in the statement of (a) is c-lineable. Trivially, every test function is bounded, has bounded gradient and is Lipschitz on $\Omega$, because it can be extended to be a test function on the whole $\mathbb{R}^{2}$. Moreover, the sum of a Lipschitz function with a non-Lipschitz one cannot be Lipschitz. Therefore $A \cap B=\varnothing$ and $A+B \subset A$, where $B:=\mathcal{C}_{c}^{\infty}(\Omega)$. An application of Lemma 2.1 (with $X=\mathcal{C}(\Omega)$ and $\mu=\mathfrak{c}$ ) yields the desired result. 
(b) It is well known that every polynomial $\mathbb{R}^{2} \rightarrow \mathbb{R}$ and every harmonic function are real-analytic (see, e.g., [8]). Therefore each function

$$
g_{n}(z):=|z|^{2 n} \varphi(z)=\left(x^{2}+y^{2}\right)^{n} \varphi(x, y) \quad(n \in \mathbb{N})
$$

is real-analytic. Here $\varphi$ is the (bounded, positive) continuous branch of $\arg z$ provided in (a) (again, we assume $z_{0}=0$ ). Recall that $\Omega$ is bounded this time. It is easily seen that each $g_{n}$ is bounded and has bounded gradient. Hence each member of the linear span of $\left\{g_{n}: n \in \mathbb{N}\right\}$ is also bounded and has bounded gradient in $\Omega$. By following the steps of (a) (but in a much easier way), one can prove that every nontrivial linear combination $F=\lambda_{1} g_{1}+\cdots+\lambda_{N} g_{N}$ is non-Lipschitz. Hence the family $A$ consisting of all functions having the properties given in the statement of (b) is lineable. Every polynomial is Lipschitz on a bounded domain and, again, the sum of a Lipschitz function with a non-Lipschitz one cannot be Lipschitz. Therefore $A \cap B=\varnothing$ and $A+B \subset A$, where by $B$ we have denoted the set of (the restrictions to $\Omega$ of) all real polynomials. Again Lemma 2.1 comes in our help (with $X=\mathcal{C}(\Omega)$ and $\mu=\operatorname{card}(\mathbb{N})$ ), so concluding the proof.

Remarks 4.3. 1. Note that since $\operatorname{dim}(\mathcal{C}(\Omega))=\mathfrak{c}$ Theorem 4.2 is optimal in terms of dimension and of number of algebra generators.

2. In the last proof, the possibility of approaching a point of a Jordan arc through two different directions has played a crucial role. Note that if the arc were not Jordan then the mentioned property would not be guaranteed. Indeed, a well known result due to E. Netto (see [12] or [14, Theorem 6.4]) asserts that a Jordan arc never fills in a space, that is, its associated curve has empty interior. Nevertheless, a Jordan arc may well be of positive bidimensional Lebesgue measure; see [14, Chapter 8].

3. The proof of Theorem 1.1 given in [11, Theorem 3.2] and [1, Theorem 6.7.1] contains a mistake, namely, the functions $f_{\lambda}(x, y)(\lambda>1)$ used there do not work as they were defined. The proof can be completed simply by replacing $y^{\lambda}$ in the expression of $f_{\lambda}$ by $|y|^{\lambda}$. We apologize for this mistake.

Acknowledgements. We are indebted to our colleague José Antonio Prado-Bassas for drawing Figure 1.

\section{REFERENCES}

[1] R. M. Aron, L. Bernal-González, D. Pellegrino, and J. B. Seoane-Sepúlveda, Lineability: The search for linearity in Mathematics, Monographs and Research Notes in Mathematics, Monographs and Research Notes in Mathematics, Chapman \& Hall/CRC, Boca Raton, FL, 2016.

[2] R. M. Aron, F. J. García-Pacheco, D. Pérez-García, and J. B. Seoane-Sepúlveda, On dense-lineability of sets of functions on $\mathbb{R}$, Topology 48 (2009), no. 2-4, 149-156.

[3] R. M. Aron, V. I. Gurariy, and J. B. Seoane-Sepúlveda, Lineability and spaceability of sets of functions on $\mathbb{R}$, Proc. Amer. Math. Soc. 133 (2005), no. 3, 795-803.

[4] R. M. Aron, D. Pérez-García, and J. B. Seoane-Sepúlveda, Algebrability of the set of nonconvergent Fourier series, Studia Math. 175 (2006), no. 1, 83-90. 
[5] A. Bartoszewicz and S. Głąb, Strong algebrability of sets of sequences of functions, Proc. Amer. Math. Soc. 141 (2013), 827-835.

[6] F. Bayart, Topological and algebraic genericity of divergence and universality, Studia Math. 167 (2005), no. 2, 161-181.

[7] L. Bernal-González, D. Pellegrino, and J. B. Seoane-Sepúlveda, Linear subsets of nonlinear sets in topological vector spaces, Bull. Amer. Math. Soc. (N.S.) 51 (2014), no. $1,71-130$.

[8] H. Cartan, Théorie élémentaire des fonctions analytiques d'une ou plusieurs variables complexes, Hermann, Paris, 1995.

[9] V. I. Gurariy and L. Quarta, On lineability of sets of continuous functions, J. Math. Anal. Appl. 294 (2004), no. 1, 62-72.

[10] P. Jiménez-Rodríguez, $c_{0}$ is isometrically isomorphic to a subspace of Cantor-Lebesgue functions, J. Math. Anal. Appl. 407 (2013), no. 2, 567-570.

[11] P. Jiménez-Rodríguez, G. A. Muñoz-Fernández, and J. B. Seoane-Sepúlveda, NonLipschitz functions with bounded gradient and related problems, Linear Algebra Appl. 437 (2012), no. 4, 1174-1181.

[12] E. Netto, Beitrag zur Mannigfaltigkeitslehre, Crelle J. 86 (1879), 263-268.

[13] W. Rudin, Real and Complex Analysis, 3rd ed., McGraw-Hill Book Co., New York, 1987.

[14] H. Sagan, Space-filling curves, Universitext, Springer-Verlag, New York, 1994.

(L. Bernal-González) Departamento de Análisis Matemático, Universidad de Sevilla, Apdo. 1160, Avenida Reina Mercedes, Sevilla, 41080 (Spain).

E-mail address: Ibernal@us.es

(P. Jiménez-Rodríguez) Department of Mathematical Sciences, Mathematics And Computer Science Building, Summit Street, Kent OH 44242, USA.

E-mail address: pjimene1@kent.edu

(G.A. Muñoz-Fernández) Departamento de Análisis Matemático, Facultad de Ciencias Matemáticas, Plaza de Ciencias 3, Universidad Complutense de Madrid, MADRID, 28040 (SPAIN).

E-mail address: gustavo_fernandez@mat.ucm.es

(J.B. Seoane-Sepúlveda) ICMAT and Departamento de Análisis Matemático, Facultad de Ciencias Matemáticas, Plaza de Ciencias 3, Universidad ComPlutense DE MADRID, MADRID, 28040 (SPAin).

E-mail address: jseoane@ucm.es 